\title{
Sprung mass positioning by semi-actively controlled damper
}

\author{
Filip Jeniš ${ }^{*}$, and Ivan Mazůrek ${ }^{1}$ \\ ${ }^{1}$ Institute of Machine and Industrial Design, Brno University of Technology, Technická 2898/2, \\ 61669 Brno
}

\begin{abstract}
Recently, the intensive wear of rails, especially in curves of small radii and at switches, has been studied. The wear is caused by the high lateral force peak of the wheel against the rail when entering the curves. An effective solution for reducing undesirable lateral forces on the rail is to rotate the vehicle bogie in the direction of the rail curve, which influences the distribution of lateral force over the first and second wheelset. This reduces the force peak and thus the track wear. The bogie rotation is nowadays realized by actuators, which replace the yaw dampers. However, actuator implementation is complicated, expensive, energyintensive and demanding for the performance of a fail-safe system. From this point of view, a semi-actively controlled yaw damper appears to be a better candidate. An algorithm such as Skyhook can hold the sprung mass in the desired position. It is believed to be possible to rotate the vehicle bogie by the special S/A control strategy of a yaw damper. This paper deals with the possibilities and limits of the positioning of the sprung mass by the semi-actively controlled damper. It has been shown that the system relative attenuation and the damper response time have the greatest influence on the mass positioning efficiency.
\end{abstract}

\section{Introduction}

Currently, high-speed rails are being developed in Europe. As train speeds increase, various problems appear, and these problems need to be solved.

Probably the biggest problem is the contradiction in the requirements for the chassis damping system when a train is being driven on a straight track at high speed and when driven through the small radius curve. While in the first case the stability achieved by high damping is decisive, in the second case, on the contrary, the damping forces must be minimized so that the bogie can rotate easily and thus minimize the lateral force of the wheel on the rail, i.e. the force which causes track wear [1]. Thus, there is a problem with contradictory requirements for dampers, which can hardly be solved with standard passive dampers.

The solution may be to use a semi-active or active damping system. The active system contains actuators instead of springs and dampers, so it can rotate the bogie at will. In the curve, it distributes a lateral force over the first and second wheelset and thus reduces the force peak [2]. However, this system is very complex, expensive, energy-intensive,

\footnotetext{
*Corresponding author: Filip.Jenis@ vutbr.cz
} 
and demanding for implementing a fail-safe system [3]. An alternative may be semi-actively controlled damping, which can change the damping force as the control unit requires.

Algorithms like Skyhook try to keep the sprung mass in the same position at all times. Using a similar algorithm, it should be possible to keep the mass in other than stable position. Stable position means a position in which the mass stabilizes under the action of a spring force and possibly a gravitational force. To deviate from this position, we need to overcome this force by the force from the damper. The damper force depends on the speed of its compression, so the idea will work only with an excitation signal of a sufficient frequency and amplitude.

The energy source for turning the chassis will be the lateral motion of the wheelset caused by its conicity, known as hunting oscillation [4]. The contribution does not deal with the emergence of this oscillation, but assumes its existence and simulates it by kinematic excitation with a sinusoidal course.

\section{Methods}

In this contribution, we consider three simplified dynamic models: a) the simplest model with 1 degree of freedom (Fig. 1, left), b) a model with 2 degrees of freedom (Fig. 1, right), c) a model with 1 degree of freedom with fixed mass $m_{2}$ (body) (Fig. 2). On the first two models, general principles will be shown. The third model simulates the movement of a train bogie.

In order to study the effects of various parameters on the behavior of mass movement efficiently, the model parameters which are entered are as general as possible. It transpired that the efficiency of the algorithm depends on the relative attenuation of the system (i.e. the ratio of weight, spring stiffness and damper coefficient), on the natural and excitation frequency ratio, on the dynamic damper range and on the time response of the damper. In more complex models then depends on the ratio of weights and on springs stiffness ratio, additionally. While maintaining these ratios, the efficiency is independent of the mass weight and the natural frequency of the system.
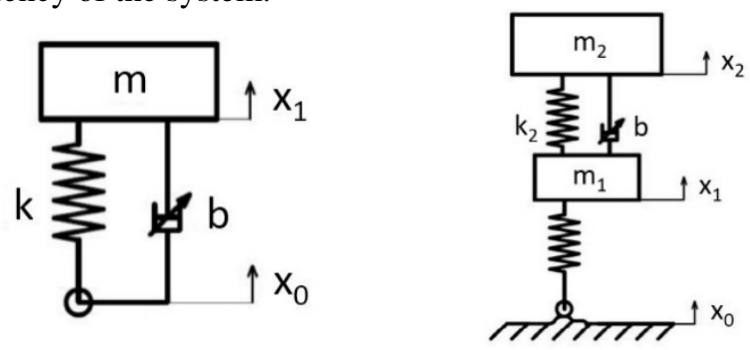

Fig. 1. Considered dynamic model, 1 DOF (left), 2 DOF (right).

\subsection{Simple 1 DOF model}

The motion equation for the one degree of freedom model is as follows:

$$
m \cdot \ddot{x}_{1}=k\left(x_{0}-x_{1}\right)+b\left(\dot{x}_{0}-\dot{x}_{1}\right)
$$

The initial values were chosen $m=100 \mathrm{~kg}$ and $f_{n}=2$. From the equations for the natural frequency and for the relative attenuation, we derived the following variables:

$$
k=4 \cdot f_{n}^{2} \cdot \pi^{2} \cdot m
$$




$$
b_{\max }=b_{r} \cdot \sqrt{m \cdot k}
$$

The dynamic range then gives the ratio of maximum and minimum damping. This equation also applies to the following models:

$$
b_{\min }=\frac{b_{\max }}{D_{r}}
$$

The damper is controlled by a semi-active algorithm, which activates high damping at the moment when the speed of the excitation movement in the positive direction is higher than the speed of the controlled mass: so at the moment when the excitation movement moves the controlled mass to the desired position. In the opposite case, the damper is deactivated. In other words, the damper is activated during compression and deactivated during expansion. Mathematically expressed:

$$
F_{b}= \begin{cases}b_{\text {max }}\left(\dot{x_{0}}-\dot{x_{1}}\right), & \left(\dot{x_{0}}-\dot{x_{1}}\right) \geq 0 \\ b_{\text {min }}\left(\dot{x_{0}}-\dot{x_{1}}\right), & \left(\dot{x_{0}}-\dot{x_{1}}\right)<0\end{cases}
$$

Where: $m$ - mass weight, $k$ - spring stiffness, $b$ - damper coefficient, $b_{\text {max }}-$ damper coefficient of high damping, $b_{\min }-$ damper coefficient of low damping, $x_{1}-$ mass displacement, $x_{0}-$ excitation signal displacement, $f_{n}$ - natural frequency, $b_{r}-$ relative attenuation, $D_{r}$ - dynamic range, $F_{b}$ - damping force.

\subsection{Model 2 DOF}

The equation and control algorithm for a model with two degrees of freedom will be similar:

$$
\begin{gathered}
m_{2} \cdot \ddot{x}_{2}=k_{2}\left(x_{1}-x_{2}\right)+b\left(\dot{x}_{1}-\dot{x}_{2}\right) \\
m_{1} \cdot \ddot{x}_{1}=k_{1}\left(x_{0}-x_{1}\right)-k_{2}\left(x_{1}-x_{2}\right)-b\left(\dot{x}_{1}-\dot{x}_{2}\right) \\
k_{2}=4 \cdot f_{n}{ }^{2} \cdot \pi^{2} \cdot m_{2} \\
b_{\text {max }}=b_{r} \cdot \sqrt{m_{2} \cdot k_{2}} \\
k_{1}=k_{2} \cdot p_{k} \\
m_{2}=m_{1} \cdot p_{m} \\
F_{b}= \begin{cases}b_{\text {max }}\left(\dot{x_{1}}-\dot{x_{2}}\right), & \left(\dot{x_{1}}-\dot{x_{2}}\right) \geq 0 \\
b_{\text {min }}\left(\dot{x_{1}}-\dot{x_{2}}\right), & \left(\dot{x_{1}}-\dot{x_{2}}\right)<0\end{cases}
\end{gathered}
$$

Where: $m_{1}$ - primary mass weight, $m_{2}$ - secondary mass weight, $p_{m}$ - mass weight ratio, $k_{1}$ - primary spring stiffness, $k_{2}-$ secondary spring stiffness, $p_{k}-$ springs stiffness ratio, $b$-damper coefficient, $b_{\max }-$ damper coefficient of high damping, $b_{\min }-$ damper coefficient of low damping, $x_{1}$ - primary mass displacement, $x_{2}$ - secondary mass displacement, $x_{0}$ - excitation signal displacement, $f_{n}$ - natural frequency, $b_{r}-$ relative attenuation, $F_{b}$ - damping force.

\subsection{DOF model of bogie rotation}

The previous two models are the basic dynamic models used for sprung and unsprung mass motion modeling. However, in the third case, we will control the bogie motion $\left(m_{l}\right)$, 
whereas we will assume that the body $\left(m_{2}\right)$ is standing because its rotation will not be allowed by the second bogie. For the purposes of this contribution, we converted the chassis model to a linear model based on the previous 2 DOF model (Fig. 2). In order to simplify model, the angular deviation can be transferred to linear $x_{l}$ and the moment of inertia $I_{l}$ corresponds to the weight $m_{l}$.

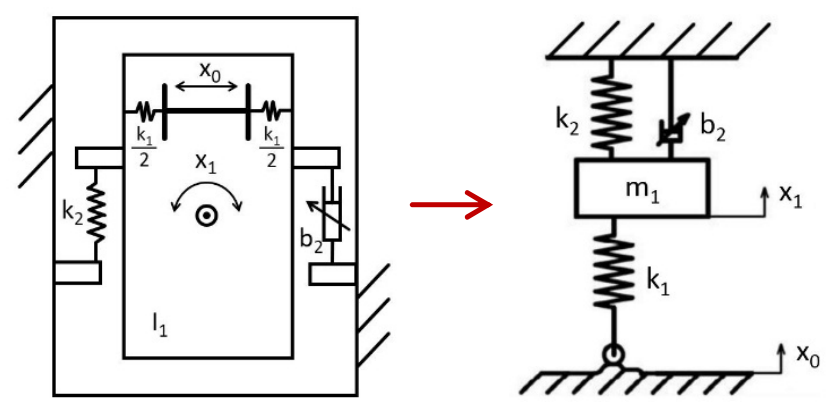

Fig. 2. Simplified dynamic model of bogie rotation.

The relevant equations are:

$$
\begin{gathered}
m_{1} \cdot \ddot{x}_{1}=k_{1}\left(x_{0}-x_{1}\right)-k_{2}\left(x_{1}\right)-b_{2}\left(\dot{x}_{1}\right) \\
k_{1}+k_{2}=4 \cdot f_{n}{ }^{2} \cdot \pi^{2} \cdot m_{1} \\
k_{1}=k_{2} \cdot p_{k} \\
b_{2 \max }=b_{r} \cdot \sqrt{m_{1} \cdot\left(k_{1}+k_{2}\right)} \\
F_{b}= \begin{cases}b_{2 \max }\left(\dot{x_{1}}\right), & \dot{x_{1}}<0 \\
b_{2 \min }\left(\dot{x_{1}}\right), & \dot{x_{1}} \geq 0\end{cases}
\end{gathered}
$$

Where: $m_{1}-$ mass weight, $k_{1}$ - prim. spring stiffness, $k_{2}-$ sec. spring stiffness, $p_{k}-$ springs stiffness ratio, $b_{2}$-damper coefficient, $b_{2 \max }$ - damper coefficient of high damping, $b_{2 \min }$ - damper coefficient of low damping, $x_{1}$ - mass displacement, $x_{0}$ - excitation signal displacement, $f_{n}$ - natural frequency, $b_{r}$ - relative attenuation, $F_{b}$ - damping force.

For all models, kinematic excitation is used - sinusoidal sweep with frequency $0-50 \cdot f_{n}$. We assume that in the train bogie case, the unsolicited wheelsets motion known as hunting motion will serve as the excitation motion. The contribution does not deal with the origin of this motion.

\section{Results}

\subsection{Simple model 1 DOF}

The graphs on fig. 3 show the dependence of the mass position on time. Over time the excitation frequency linearly increases. We can see that in the resonance area (excitation frequency/natural frequency $=1$ ) the algorithm does not work and the mass oscillates. Above 
the system resonant frequency, the mass motion is already stable, so we see how many times
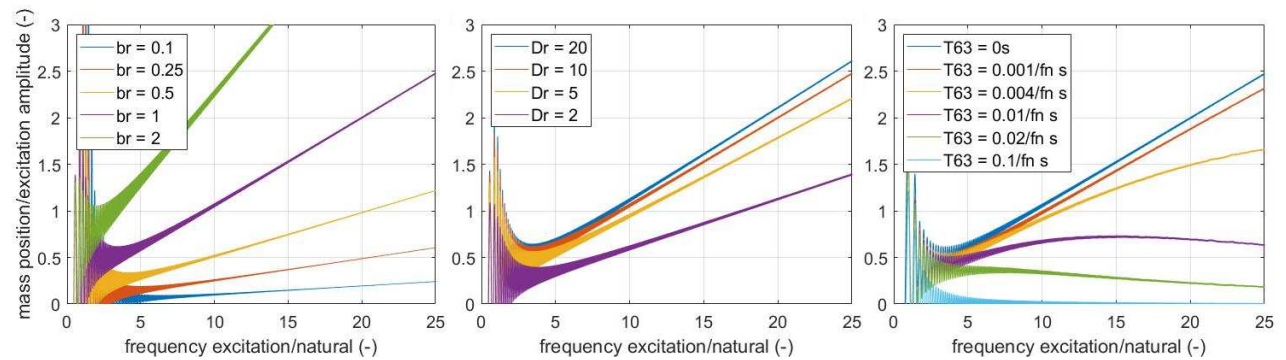

Fig. 3. Dependence of achieved deviation on excitation frequency for various parameters of: a) relative attenuation, b) dynamic range, c) response time for 1 DOF model. Parameters that are not invariable are $b_{r}=1, D_{r}=10, \tau_{63}=0$.

the achieved position of mass will be higher than the amplitude of the excitation motion, depending on the ratio of the excitation frequency and the system natural frequency. The results from simulations showed the influence of the system relative attenuation $\left(b_{r}\right)$, the dynamic range $\left(D_{r}\right)$ and the response time of the damper $\left(\tau_{63}\right)$. The frequency dependence is linear, and efficiency increases with increasing relative attenuation, i.e. the maximum damping force of the damper. For commonly achieved dynamic ranges around 10 [5], the method works well. Further increase of the dynamic range does not make much sense. However, the function can be significantly impaired by the large response time of the damper. For reasonable efficiency, it needs to be less than $0.004 / f n s$.

\subsection{Model 2 DOF}

In the two degrees of freedom model, the same influences were investigated and additionally influenced on the mass weight ratio and springs stiffness ratio. Figure 4 show that the efficiency course here is not linearly dependent on the excitation frequency, but the highest efficiency is in the area of the natural frequency of mass $m_{l}$. The dependence on the relative attenuation and dynamic range is similar to the previous case, with the efficiency in the area of $m_{1}$ natural frequency being approximately the same, but decreasing rapidly above the resonant frequency for low relative attenuations. Since the highest efficiency is achieved at lower frequencies, there is not such a significant effect of response time. A response time of at least $0.02 / f_{n}$ is required for effective control. The maximum efficiency does not depend on the mass ratio, but for low ratios $m_{2} / m_{1}$ with increasing resonant frequency, the efficiency decreases rapidly. The efficiency is strongly dependent on the $k_{1} / k_{2}$ ratio. The larger the $k_{1}$ compared to $k_{2}$, the higher position of the monitored mass. Using the usual parameters, it should be possible to deviate the mass to approximately 3.5 times the amplitude of the excitation motion.
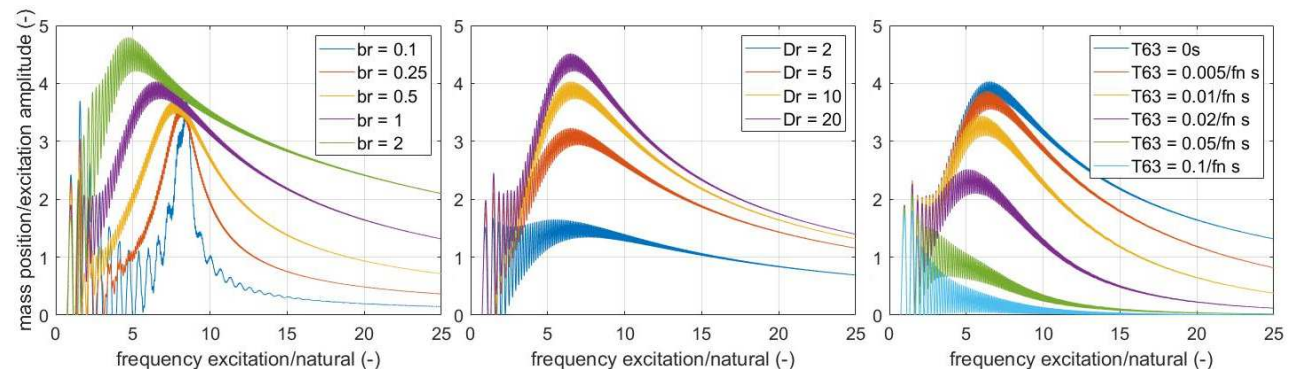

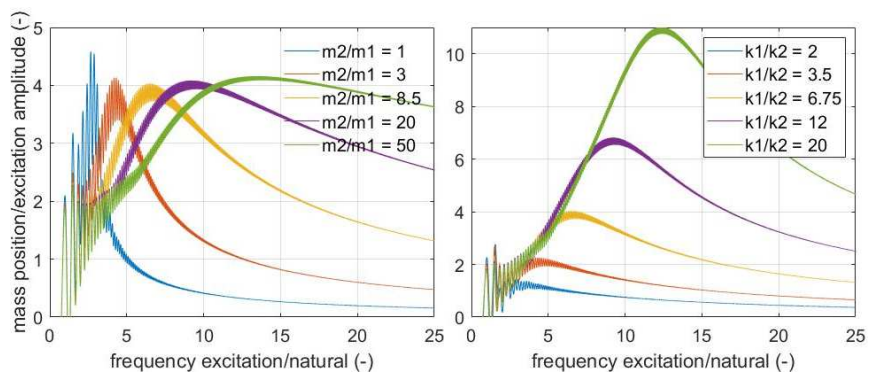

Fig. 4. Dependence of achieved deviation on excitation frequency for various parameters of: a) relative attenuation, b) dynamic range, c) time response, d) $m_{1} / m_{2}$ ratio, e) $k_{1} / k_{2}$ for 2 DOF model. Parameters that are not invariable are $b_{r}=1, D_{r}=10, \tau_{63}=0, m_{2} / m_{1}=8.5, k_{1} / k_{2}=6.75$.

\subsection{Model 1 DOF of bogie rotation}

The same influences were investigated in the last model (Fig. 5). Unlike the previous models, the efficiency here is conditioned by a significantly greater relative attenuation. To make the algorithm work, the system must be damped supercritically, and even then it does not reach such high values as in the previous cases. The influences of other parameters remain similar. The efficiency increases with a dynamic range up to about $D_{r}=10 . \tau_{63}=0.01 / f_{n} s$ could be considered as a sufficiently short response time. And as in the previous case, the stiffness of $k_{1}$ needs to be as large as possible compared to $k_{2}$. There is no clear maximum possition on the resonant frequency as in case of 2 DOF model; however, with increasing excitation frequency, the efficiency also decreases.
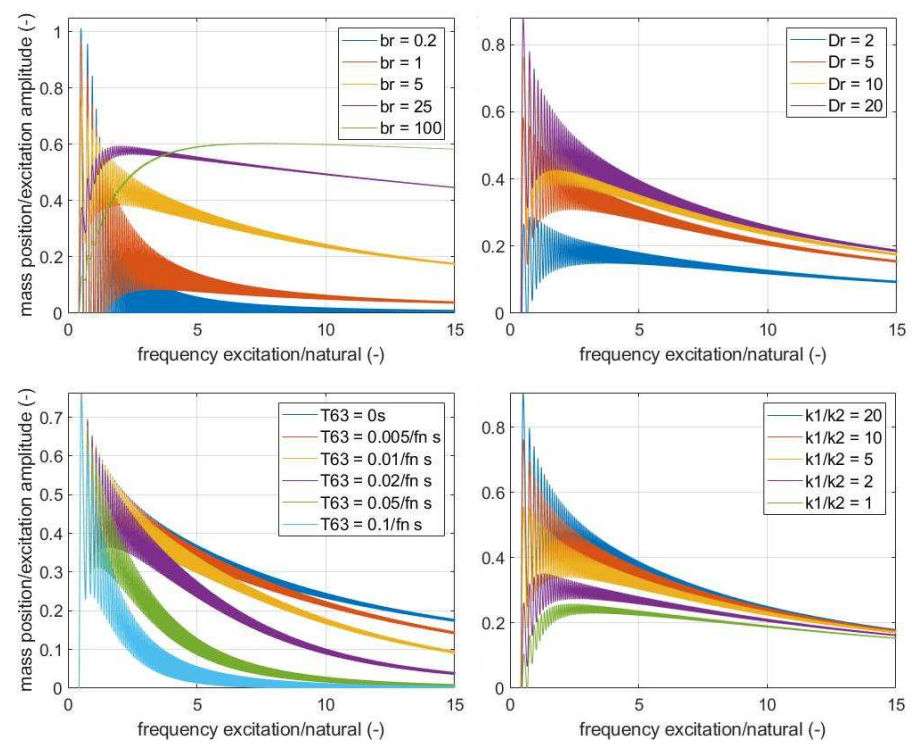

Fig. 5. Dependence of achieved deviation on excitation frequency for various parameters of: a) relative attenuation, b) dynamic range for 1 DOF model of bogie rotation, c) time response d) $k_{1} / k_{2}$ ratio for $1 \mathrm{DOF}$ model of bogie rotation. Parameters that are not invariable are $b_{r}=5, \underline{\underline{D_{r}}}=10, \tau_{63}=0$, $k_{1} / k_{2}=6.75$. 


\section{Conclusion}

This contribution deals with the possibility of positioning the sprung mass using a semiactively controlled damper. The phenomenon is studied on three simple models. The relative attenuation of the system and the response time proved to have the greatest influence on the effectiveness of the strategy. For efficient control, it is suitable that $b_{r}>1$, i.e. that the system is attenuated supercritically. We consider $0.01 / f_{n} \mathrm{~s}$ to be a sufficient response time. Strecker et al. designed a damper with a response time of $1.6 \mathrm{~ms}$ [6], which would be enough to control a system with a natural frequency of $6 \mathrm{~Hz}$.

A simple 1 DOF model achieves the best results, but unfortunately, it is not applicable on train chassis control. The phenomenon verified on the 2 DOF model could be well used for positioning the train body as a secondary sprung mass, for example for tilting it into curves. This simplified model is also often used in the design of automotive suspension, here a semi-active damper could be used to change the ground clearance, for which today hydropneumatic or other suspensions are used [7]. These hypotheses are currently being tested.

The effectiveness of the strategy is highly dependent on the location of the semi-active controlled damper; in other words, it is dependent on which mass is controlled. In the first two models, the damper is located between the controlled mass and the mass performing the excitation motion - thus in the desired direction (during compression) it can easily serve to transfer the energy of the excitation motion to the controlled mass. In the opposite direction (when rebounding), energy transfer is minimal. A sufficient supply of energy is necessary to overcome the force of the spring and thus to keep the mass in the desired position. If the damper in this place is conventional, the energy transfer will be the same when moving in both directions.

The disadvantage of the third model is that the controlled damper is located between the controlled mass and the fixed mass. So, it can only try to prevent the mass from moving in the undesired direction, and not prevent it from moving in the opposite direction, and its ability for ensuring the transfer of energy from the excited mass is limited. It is usual for semi-active algorithms not to work well with the original (passive) system parameters, but for higher efficiency, these parameters must be modified. In the first place, the damper force in the activated state compared to the passive damper must be increased. We can see the same trend in this case as well. The effectiveness of the strategy increases mainly with a relative attenuation of the system. It means that for satisfactory results we need to increase the damper force significantly, or reduce the stiffness of the springs, especially $k_{2}$, which acts against the controlled damper. An increase in the $k_{1} / k_{2}$ ratio will also have an important effect. However, these changes will not be possible without structural modification in the train chassis.

\section{Acknowledgement}

The authors wish to acknowledge the support of the grant nr PPI//APM/2018/1/00027/ DEC/1, National Agency for Academic Exchange, Poland, and FEKT/FSI-J-20-6260, and FSI-S-20-6247

\section{References}

1. G. Diana, S. Bruni, et. al., Vehicle Syst. Dyn. 37, 3 (2002)

2. F. Braghin, S. Bruni, F. Resta, Vehicle Syst. Dyn. 44, 11 (2006)

3. X. Wei, M. Zhu, L. Jia, Vehicle Syst. Dyn. 54, 7 (2016) 
4. A. H. Wickens. Int. J. Solids Struct. 1, 3 (1965)

5. O. Macháček, M. Kubík, et. al., Adv. Mech. Eng. 11, 3 (2019)

6. Z. Strecker, M. Kubik, et. al., Smart Mater. Struct. 28, 5 (2019)

7. N. Amati, A. Tonoli, et. al., P. I. Mech. Eng. D-J Aut. 232, 9 (2018) 